\title{
AN ALGEBRAIC CLASSIFICATION OF SOME EVEN-DIMENSIONAL SPHERICAL KNOTS. I
}

\author{
BY
}

M. S. FARBER

\begin{abstract}
The main result of the paper is the classification of simple even-dimensional spherical knots in terms of their algebraic invariants.
\end{abstract}

This is the first of two papers whose aim is to give a complete solution of the classification problem of simple even-dimensional spherical knots of codimension two in terms of the algebraic invariants of the complement's infinite cyclic covering. In this first article the problem will be reduced to the problem of classifying certain algebraic objects, called $P$-quintets, with respect to $R$-equivalence. In the second article the algebraic problem thus obtained will be solved in the sense that to each $P$-quintet we shall assign a new algebraic object, called a $\Lambda$-quintet, two $P$-quintets being $R$-equivalent if and only if the corresponding $\Lambda$-quintets are isomorphic. The modules which constitute the $\Lambda$-quintet will then be interpreted in terms of the invariants of the infinite cyclic covering of the complement of the knot under consideration. The final results in the case of fibred knots were given in $[6,8]$ and in the case of spherical knots were announced in subsection 8.13 of [8].

Let us briefly describe the history of the subject.

In $1970 \mathrm{~J}$. Levine [14] gave an algebraic classification of simple odd-dimensional spherical knots. He showed that the type of such knots is determined by the Seifert matrix, considered up to $S$-equivalence. Later in the works of Trotter [20] and Kearton [10] this same class of knots was described in a more invariant way in terms of Alexander modules and Blanchfield pairings.

These fundamental papers gave grounds to hope that the algebraic description of the class of simple even-dimensional spherical knots-the next class in order of difficulty after Levine's simple odd-dimensional knots - could be obtained by similar methods, based on the direct isotopy of handle decompositions. This hope was not entirely fulfilled, although certain partial results were obtained. The main difficulty in this direction was the description of the obstructions to the isotopy of Seifert manifold handles in the case when the knot module possesses 2-torsion. In the absence of 2-torsion, Kearton [11] gave an algebraic classification. However, the relationship between the algebraic object constructed in [11] and the classical knot invariants, e.g. the Alexander modules, remained unclear. Even more particular (but

Received by the editors November 30, 1982.

1980 Mathematics Subject Classification. Primary 57C45; Secondary 55D15.

(C)1984 American Mathematical Society $0002-9947 / 84 \$ 1.00+\$ .25$ per page 
clearer) results were obtained by Kojima [12], who considered knots with torsion-free Alexander modules.

In 1974-1975, in the works of J. Levine $[\mathbf{1 5}, \mathbf{1 6}]$ and the author $[\mathbf{3 , 4}]$ (also see the Soviet review journal Matematika, 1974,6A675 and 9A640), new pairings ${ }^{1}$ defined on the torsion subgroups of the Alexander modules, with values in $\mathbf{Q} / \mathbf{Z}$, were constructed. One of these pairings is defined on the middle-dimensional Alexander module of an even-dimensional knot. In many cases in the study of even-dimensional knots (see [4, 16]) this pairing plays the same role as the Blanchfield pairing in the study of odd-dimensional knots, and also must appear in the algebraic description of simple even-dimensional knots. As shown by Kojima [13], in the case when the Alexander module is finite and has no 2-torsion, this pairing, together with the Alexander module, determines the knot type entirely. However, as will follow from the results of this paper, in the general case additional algebraic invariants are needed. In his paper Kojima also applied methods similar to those of J. Levine.

An entirely new possibility for obtaining algebraic descriptions of knots arose in view of the papers [5, 7], where a classification of a fairly wide class of knots-stable spherical knots - was obtained in homotopic terms. This class of knots contains both Levine's simple knots and simple even-dimensional knots. Generally speaking, in order to obtain the algebraic classification from the homotopy one, it is necessary to have an algebraic description of stable homotopy type and a complete set of algebraic invariants determining the homotopy class of a continuous map. Although a complete solution of this problem is not provided by algebraic topology, in order to study certain subsets of the set of stable knots it is necessary to solve this problem for the case of special classes of spaces and maps. For example, the case of Levine knots corresponds to the study of $(q-1)$-connected complexes of dimension $\leqslant q$ and their maps; clearly this space has the stable homotopy type of a wedge of $q$-dimensional spheres and the stable homotopy class of maps of such spaces is determined by the induced homomorphism of $q$-dimensional homology. The case of simple even-dimensional knots corresponds to a more difficult homotopy problem which was solved in [8] (note that well-known methods of algebraic topology give the solution of the problem considered in $\$ \S 6$ and 7 of [8] in the form of two successive obstructions, which is unsatisfactory for our purpose).

The method outlined above for obtaining the algebraic classification of knots was carried out in [8], where it yielded the algebraic classification (modulo-finiteness) of all stable fibered knots, as well as the complete algebraic classification of simple even-dimensional fibered knots. The same outline is followed in the present paper.

Its plan is the following. In $\$ 1$ we provide certain facts from stable homotopy theory in a form convenient for further use. In $\$ 2$ we introduce the notion of isometry, define the $R$-equivalence of isometries and give the classification of stable knots in terms of isometries. These results are essentially a restatement of the results of $[5,7]$, but are given in a form more convenient for algebraic classification, the

\footnotetext{
'Although the constructions proposed by J. Levine and the author are different, it is easy to show that they yield the same pairings.
} 
$R$-equivalence relation being presented in a more algebraic form. Further, in $\S 3$, the homotopy results of $[\mathbf{8}]$ are applied to the study of dualized spaces. In the conclusive $\S 4$ the results of all the previous sections are amalgamated, and this gives the algebraic classification of simple even-dimensional spherical knots.

In conclusion, a few words on terminology: An $n$-dimensional knot is a pair $\left(S^{n+2}, k^{n}\right)$, consisting of an oriented sphere $S^{n+2}$ and its closed smooth oriented $n$-dimensional submanifold $k$. A knot is called spherical if $k$ is a homotopy sphere. Two knots $\left(S^{n+2}, k_{1}^{n}\right)$ and $\left(S^{n+2}, k_{2}^{n}\right)$ are said to be equivalent (or of the same isotopy type) if there exists a smooth isotopy of the sphere $S^{n+2}$ sending $k_{1}$ onto $k_{2}$ with preserved orientations.

A knot $\left(S^{n+2}, k^{n}\right)$ is called $r$-simple if $\pi_{i}\left(S^{n+2}-k\right) \approx \pi_{i} S^{1}$, when $i \leqslant r$ [10]. An $n$-dimensional knot is called stable if it is $([n / 3]+1)$-simple and $n \geqslant 5$ [6]. A simple even-dimensional knot is a $(q-1)$-simple knot of dimension $2 q$ (see [11]).

1. The stable homotopy category. It was established in $[5,7]$ that the classification problem of stable knots reduces to certain problems in stable homotopy theory. In $\$ 2$ of the present paper these problems of stable homotopy will be somewhat reformulated. In the present section we describe the stable homotopy category most conveniently for this purpose.

This category will be denoted by $\mathrm{Stab}_{0}$. It is a full subcategory of the category Stab introduced by A. Dold and D. Puppe [1].

1.1. Objects of the category $\operatorname{Stab}_{0}$ are pairs of the form $(X, n)$, where $X$ is a finite $\mathrm{CW}$-complex with base point and $n \in \mathbf{Z}$ is an integer. The set of morphisms from $(X, n)$ to $(Y, m)$, denoted further by figure brackets $\{$,$\} is by definition the direct$ limit

$$
\lim _{k \rightarrow \infty}\left[S^{n+k} \wedge X, S^{m+k} \wedge Y\right]
$$

where $\wedge$ is the usual smash product, the square brackets [, ] denoting the set of homotopy classes and the bonding maps

$$
\left[S^{n+k} \wedge X, S^{m+k} \wedge Y\right] \rightarrow\left[S^{n+k+1} \wedge X, S^{m+k+1} \wedge Y\right],
$$

appearing in the definition of the direct limit, are given by smashing with $S^{1}$. The composition of morphisms is defined in an obvious way.

The category $\mathrm{Stab}_{0}$, as well as $\mathrm{Stab}$, is an additive one. Hence the usual constructions for additive categories e.g. direct sums, may be carried out in it [17]. Any two objects of $\mathrm{Stab}_{0}$ have a direct sum.

The functors $\Sigma^{p}, p \in \mathbf{Z}$, where $\Sigma^{p}(X, n)=(X, n+p)$ are defined in the category $\mathrm{Stab}_{0}$. Clearly $\Sigma^{p} \circ \Sigma^{q}=\Sigma^{p+q}$ and $\Sigma^{0}$ is the identity functor.

Pairs of the form $(X, 0)$ are usually simply written as $X$. Then we can write $\Sigma^{n} X$ instead of $(X, n)$. Note that for $n \geqslant 0$ the object $\Sigma^{h} X$ is equivalent in $\operatorname{Stab}_{0}$ to the $n$-fold suspension over $X$.

The objects of the category $\mathrm{Stab}_{0}$ will be called virtual complexes and the morphisms $S$-maps. Virtual complexes equivalent to complexes of the form $\Sigma^{n} X$ with $n \geqslant 0$ will be called true. 
1.2. Now we shall describe, still following [1], a monoid structure of the category $\mathrm{Stab}_{0}$.

On the set of virtual complexes we have the tensor product operation

$$
(X, n) \otimes(Y, m)=(X \wedge Y, n+m) .
$$

We can also define the tensor product of $S$-maps: if two $S$-maps

$$
(X, n) \rightarrow\left(X^{\prime}, n^{\prime}\right) \text { and }(Y, m) \rightarrow\left(Y^{\prime}, m^{\prime}\right)
$$

are presented by the maps

$$
f: S^{n+k} \wedge X \rightarrow S^{n^{\prime}+k} \wedge X^{\prime}, \quad g: S^{m+l} \wedge Y \rightarrow S^{m^{\prime}+l} \wedge Y^{\prime},
$$

respectively, then their tensor product is obtained by taking the map

$$
f \wedge g: S^{n+k} \wedge X \wedge S^{m+l} \wedge Y \rightarrow S^{n^{\prime}+k} \wedge X^{\prime} \wedge S^{m^{\prime}+l} \wedge Y^{\prime}
$$

and transforming it into the map

$$
S^{n+m+k+l} \wedge X \wedge Y \rightarrow S^{n^{\prime}+m^{\prime}+k+l} \wedge X^{\prime} \wedge Y^{\prime}
$$

by interchanging the middle terms and multiplying the homotopy class thus obtained by $(-1)^{\left(n+n^{\prime}\right) l}$. Then $\otimes$ will be a bifunctor $\operatorname{Stab}_{0} \times \operatorname{Stab}_{0} \rightarrow \operatorname{Stab}_{0}$ covariant and additive in both variables.

The commutativity of the tensor product is expressed by the existence of the equivalence

$$
\gamma:(X, n) \otimes(Y, m) \rightarrow(Y, m) \otimes(X, n),
$$

which is defined as the $S$-homotopy class of the usual interchange map $X \wedge Y \rightarrow Y$ $\wedge X$ multiplied by $(-1)^{m n}$.

The associativity is expressed by the existence of the obvious natural equivalence

$$
((X, n) \otimes(Y, m)) \otimes(Z, k) \rightarrow(X, n) \otimes((Y, m) \otimes(Z, k)) .
$$

There is also the identity object $I=\left(S^{0}, 0\right)$, for which there are natural equivalences

$$
I \otimes(X, n) \rightarrow(X, n), \quad(X, n) \otimes I \rightarrow(X, n) .
$$

1.3. For any extraordinal homology theory $h_{*}$ on the category of finite based complexes, we can put $h_{i}(X, n)=h_{i-n}(X)$ and define the action on morphisms in an obvious way. As a result, we obtain a homology theory on $\mathrm{Stab}_{0}$, and similarly for cohomology.

1.4. A virtual complex $\mathfrak{X}=(X, n)$ will be called $r$-connected, where $r \in \mathbf{Z}$, if $H_{i}(\mathcal{X})=0$ for $i \leqslant r$. The largest such number $r$ is called the connectivity of $\mathcal{X}$ and denoted by conn $X$.

We shall say that the dimension of $\mathfrak{X}$ is no greater than $m$ if $H^{i}(\mathfrak{X})=0$ for $i>m$. The smallest such number $m$ will be called the dimension of $\mathcal{X}$ and denoted by $\operatorname{dim} \mathfrak{X}$.

The number $\operatorname{dim} \mathfrak{X}-\operatorname{conn} \mathfrak{X}$ will be denoted by length $\mathcal{X}$ and called the length of $\mathcal{X}$. For zero objects of the category $\operatorname{Stab}_{0}$ (i.e. for objects $(X, n)$ where $X$ has the stable homotopy type of a point), we will agree that the connectivity equals $+\infty$, the dimension equals $-\infty$, and the length equals $-\infty$. For nonzero objects $\mathscr{X}$, the dimension and connectivity are finite and length $\mathscr{X}>0$. 
1.5. Lemma. If $X$ is a true finite complex for which length $X \leqslant \operatorname{conn} X=1$, then $X$ is stable homotopy equivalent to the suspension over some finite true complex.

This is a standard fact (see [18, Chapter 8, exercise D1]). Its proof may be easily obtained from this exercise.

Lemma 1.5 implies

1.6. Corollary. Any virtual complex $\mathcal{X}$ with length $\mathcal{X} \leqslant \operatorname{conn} \mathcal{X}+2$ is a true complex.

Proof. Suppose $\mathcal{X}=\Sigma^{n} X$. We may assume that $n<0$, since in the converse case the statement is obvious. Since conn $X=\operatorname{conn} \mathcal{X}-n$, length $X=$ length $\mathcal{X}$, the assumptions of the corollary are equivalent to length $X \leqslant$ conn $X+n+2$. Since $n<0$ we can apply Lemma 1.5 , so that $X \sim S X_{1}$. Then

$$
\text { conn } X_{1}=\text { conn } X-1 \text {, length } X_{1}=\text { length } X
$$

and

$$
\text { length } X_{1} \leqslant \text { conn } X_{1}+n+1+2 \text {. }
$$

If $n+1<0$, then we can use Lemma 1.5 again, obtaining the complex $X_{2}$ satisfying $S X_{2} \sim X_{1}$ or $S^{2} X_{2} \sim X$. Continuing in this manner, we will get a complex $X_{-n}$ for which $S^{-n} X_{-n} \sim X$. But then $\mathcal{X}$ is equivalent in $\mathrm{Stab}_{0}$ to the complex $X_{-n}$, which proves the corollary.

One of the most important properties of the category $\mathrm{Stab}_{0}$ is the following theorem on the splitting of idempotents.

1.7. TheOREM. Suppose $f: \mathcal{X} \rightarrow \mathfrak{X}$ is a morphism in the category $\mathrm{Stab}_{0}$ such that $f^{2}=f$. Then there exists a virtual complex $\mathcal{Y}$ and morphisms $i: \mathcal{O} \rightarrow \mathcal{X}$ and $\pi: \mathcal{X} \rightarrow \mathcal{Y}$ such that $\pi \circ i=1_{\text {y }}$ and $i \circ \pi=f$.

This theorem was proved by P. Freyd [9]. The more general nonstable case was studied by D. Edwards and R. Geoghegan [2].

2. Isometries and knots. The purpose of this section is to describe the classification of stable knots in terms of isometries over the stable homotopy category $\mathrm{Stab}_{0}$. In $[5,7]$ the classification of such knots is described in the language of Seifert homotopy pairings. The passage to the isometry language may be carried out by using the splitting idempotents theorem.

2.1. An $n$-isometry is a triplet $(\mathfrak{X}, u, z)$ consisting of a virtual complex $\mathscr{X}$ and $S$-maps $u: \mathfrak{X} \otimes \mathfrak{X} \rightarrow S^{n+1}, z: \mathfrak{X} \rightarrow \mathfrak{X}$, satisfying the following conditions:

(a) $u$ is a Spanier-Whitehead duality,

(b) $u^{\prime}=(-1)^{n+1} u$,

(c) $u \circ\left(z \otimes 1_{\mathscr{x}}\right)=u \circ\left(1_{\mathfrak{X}} \otimes \bar{z}\right)$.

Here $u^{\prime}$ is (by definition) $u \circ \gamma$, where $\gamma$ was defined in subsection 1.2 and $\bar{z}=1_{\mathfrak{X}}-z$. Recall that condition (a) means that for all virtual complexes $\mathscr{Q}$ and $\mathscr{B}$ the map

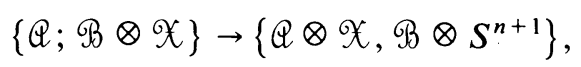

sending every morphism $f: \mathbb{Q} \rightarrow \Re \otimes \mathscr{X}$ into the morphism $\left(1_{\mathscr{B}} \otimes u\right) \circ\left(f \otimes 1_{\mathfrak{X}}\right)$, is an isomorphism (see [1, 19]). 
REMARK. In [6] we already used the notion of $n$-isometry in a more restricted sense. Indeed, if in the notation of $[6, \mathrm{p} .108]$, we put $z=(1-t)^{-1}$, it is easy to see that conditions (a), (b), (c) will be satisfied. Conversely, if in addition to these conditions we require that $\mathcal{X}$ be a true complex and $z$ be an $S$-equivalence, then we can put $t=1-z^{-1}$, where $z^{-1}$ is the inverse object to $z$ in $\operatorname{Stab}_{0}$ and we get the definition from [6, p. 108].

2.2. Two $n$-isometries $\left(\mathfrak{X}_{\nu}, u_{\nu}, z_{\nu}\right), \nu=1,2$, are called congruent, if there exists an $S$-equivalence $f: \mathfrak{X}_{1} \rightarrow \mathfrak{X}_{2}$ for which $u_{2} \circ(f \otimes f)=u_{1}$ and $z_{2} \circ f=f \circ z_{1}$. Two isometries $\left(\mathfrak{X}_{\nu}, u_{\nu}, z_{\nu}\right), \nu=1,2$, will be called contiguous if there exist $S$-maps $\varphi$ : $\mathcal{X}_{1} \rightarrow \mathcal{X}_{2}$ and $\psi: \mathcal{X}_{2} \rightarrow \mathcal{X}_{1}$ such that

(I) $u_{2} \circ\left(1_{x_{2}} \otimes \varphi\right)=u_{1} \circ\left(\psi \otimes 1_{x_{1}}\right)$,

(II) $z_{2} \circ \varphi=\varphi \circ z_{1}, z_{1} \circ \psi=\psi \circ z_{2}$,

(III) $\psi \circ \varphi=z_{1} \circ \bar{z}_{1}, \varphi \circ \psi=z_{2} \circ \bar{z}_{2}$.

Recall that here, as above, $\bar{z}_{1}=1-z_{1}, \bar{z}_{2}=1-z_{2}$.

Congruent $n$-isometries are contiguous. Indeed, if $f$ is the equivalence, appearing in the definition of congruence, and $g$ is the inverse one, then we can put $\varphi=z_{2} \circ f$, $\psi=\bar{z}_{1} \circ g$, and all the conditions from the definition of contiguity will hold.

Note that in the statement of the definition of contiguity some conditions are superfluous. For example, each of the two relations in (II) is a consequence of the other one and relation (I). Similarly, each of the two relations in (III) is a consequence of the other one and (I). The statement given above expresses the fact that the contiguity relation is symmetric.

Contiguity, unlike congruence, is not an equivalence relation since it is not transitive. The equivalence relation generated on the set of all $n$-isometries by the contiguity relation will be called $R$-equivalence.

2.3. Every $n$-isometry $(\mathfrak{X}, u, z)$ determines the $(n+4)$-isometry $\left(\Sigma^{2} \mathfrak{X}, v, \Sigma^{2} z\right)$, where $v$ is the composition

$$
\begin{aligned}
& \Sigma^{2} \mathfrak{X} \otimes \Sigma^{2} \mathfrak{X}=\left(\Sigma^{2} S^{0} \otimes \mathfrak{X}\right) \otimes\left(\Sigma^{2} S^{0} \otimes \mathfrak{X}\right) \rightarrow \Sigma^{2} S^{0} \otimes \Sigma^{2} S^{0} \otimes \mathfrak{X} \otimes \mathfrak{X} \\
& \stackrel{1 \otimes 1 \otimes 0}{\rightarrow} \Sigma^{2} S^{0} \otimes \Sigma^{2} S^{0} \otimes S^{n+1} \approx S^{n+5}
\end{aligned}
$$

the first arrow denoting the interchange (in the sense of 1.2) of the middle factors, the second one being $1 \otimes 1 \otimes u$. It is easy to check that $\left(\Sigma^{2} \mathfrak{X}, v, \Sigma^{2} z\right)$ is indeed an isometry. It will be called double suspension over the given isometry and denoted by $\Sigma^{2}(\mathfrak{X}, u, z)$.

Note that a similar construction on $\Sigma \mathfrak{X}$ does not yield an $(n+2)$-isometry since condition (b) from 2.1 will not be satisfied.

Two $n$-isometries are congruent (contiguous or $R$-equivalent) if and only if their double suspensions are congruent (respectively, contiguous or $R$-equivalent). Therefore classes of $n$-isometries with respect to congruence and with respect to $R$-equivalence only depend on the residue of $n$ modulo 4 .

Also note that the double suspension operation does not change the length of $\mathscr{X}$, but increases the connectivity by 2 . By Corollary 1.6 , this means that an iteration of the double suspension gives an isometry defined on a true complex. 
2.4. The pair $(\mathcal{X}, \theta)$, where $\mathscr{X}$ is a virtual complex and $\theta: \mathcal{X} \otimes \rightarrow S^{n+1}$ is an $S$-map, is called an $n$-pairing. An $n$-pairing is called spherical, if $\theta+(-1)^{n+1} \theta^{\prime}$ is a duality. Here $\theta^{\prime}=\theta \circ \gamma$, where $\gamma$ was defined in subsection 1.2.

Any $n$-isometry ( $\mathfrak{X}, u, z)$ determines a spherical pairing $(\mathfrak{X}, \theta)$, where $\theta=u \circ(1$ $\otimes z$ ). Let us check that $\theta$ is spherical:

$$
\begin{aligned}
\theta+(-1)^{n+1} \theta^{\prime} & =u \circ(1 \otimes z)+(-1)^{n+1} u^{\prime} \circ(z \otimes 1) \\
& =u \circ(1 \otimes z)+u \circ(z \otimes 1)=u \circ(1 \otimes z)+u \circ(1 \otimes \bar{z})=u,
\end{aligned}
$$

which is a duality. Here we first used the property (b), then (c) and finally (a) (see 2.1).

Conversely, every spherical $n$-pairing $(\mathfrak{X}, \theta)$ determines an $n$-isometry $(\mathfrak{X}, u, z)$, where $u=\theta+(-1)^{n+1} \theta^{\prime}$ and $z: \mathcal{X} \rightarrow \mathcal{X}$ is determined from the relation $u \circ(1 \otimes z)$ $=\theta$ (since $u$ is a duality, this condition completely defines $z$ ). Then the properties (a) and (b) of subsection 2.1 will hold trivially, while property (c) is proved as follows: transposing terms in $u \circ(1 \otimes z)=\theta$ and using (b) we get $u \circ(z \otimes 1)=(-1)^{n+1} \theta^{\prime}$; now adding these two relations we find

$$
u \circ(1 \otimes z)+u \circ(z \otimes 1)=u,
$$

which obviously implies (c).

Thus, spherical n-pairings and n-isometries are in one-to-one correspondence.

Any $n$-pairing $(\mathfrak{X}, \theta)$ determines an $(n+2)$-pairing $\left(\Sigma \mathcal{X}, \theta_{1}\right)$, where $\theta_{1}$ is the composition

$$
\begin{aligned}
& \Sigma \mathscr{X} \otimes \Sigma \mathfrak{X}=\left(\Sigma S^{0} \otimes \mathfrak{X}\right) \otimes\left(\Sigma S^{0} \otimes \mathfrak{X}\right) \rightarrow \Sigma S^{0} \otimes \Sigma S^{0} \otimes \mathfrak{X} \otimes \mathfrak{X} \\
& \stackrel{1 \otimes 1 \otimes \theta}{\rightarrow} \Sigma S^{0} \otimes \Sigma S^{0} \otimes S^{n+1} \approx S^{n+3},
\end{aligned}
$$

in which the first arrow is the interchanges $S$-map described in 1.2. This pairing is said to be the suspension of the given pairing and is denoted by $\Sigma(\mathfrak{X}, \theta)$. It is easy to see that the suspension of a spherical pairing is not necessarily spherical. However, the double suspension $\Sigma^{2}(\mathfrak{X}, \theta)$ preserves sphericity.

2.5. Suppose $K=\left(S^{n+2}, k^{n}\right)$ is an $n$-dimensional $r$-simple spherical knot and let $V^{n+1} \subset S^{n+2}$ be an $r$-connected Seifert manifold of this knot. Then as shown in $[5,7]$ the Seifert homotopy pairing $\theta: V \wedge V \rightarrow S^{n+1}$ defines a spherical $n$-pairing $(V, \theta)$. The $n$-isometry $(V, u, z)$ corresponding to this $n$-pairing will be called the $n$-isometry of the manifold $V$.

2.6. ThEOREM. The R-equivalence class of the n-isometry $(V, u, z)$ does not depend on the choice of the Seifert manifold $V$ and is well defined by the type of the given spherical knot. If $3 r \geqslant n+1 \geqslant 6$, then the map, sending each knot to the R-equivalence class of the n-isometry of some r-connected Seifert manifold of this knot, is a bijection of the set of isotopy types of r-simple $n$-dimensional spherical knots on the set of $R$-equivalence classes of $n$-isometries given on all virtual complexes of length $\leqslant n-2 r$.

For the proof, we shall show that this theorem is a restatement of the main theorem in $[5,7]$. To do this, we shall first find a relation on the set of spherical 
pairings which corresponds to continguity on the set of isometries with respect to the bijection described in 2.4 (this relation will also be called contiguity), and then compare the set of classes of $R$-equivalence with a similar set constructed only on true complexes and used in $[5,7]$. The proof of Theorem 2.6 will be concluded in subsection 2.11.

2.7. Suppose $X_{1}$ and $X_{2}$ are two virtual complexes and $u$ : $X_{1} \otimes X_{2} \rightarrow S^{n+1}$ is a duality. Then the $S$-map $\theta$ : $\mathfrak{X} \otimes \mathfrak{X} \rightarrow S^{n+1}$, where $\mathfrak{X}=\mathfrak{X}_{1} \oplus \mathfrak{X}_{2}$, while $\theta$ is given by the matrix $\left(\begin{array}{ll}0 & u \\ 0 & 0\end{array}\right)$, determines a spherical $n$-pairing $(\mathfrak{X}, \theta)$. The $n$-pairings congruent to $n$-pairings of this type will be called special.

Suppose $\left(\mathfrak{X}_{\nu}, \theta_{\nu}\right)$ are two $n$-pairings, $\nu=1,2$. They are called contiguous (compare $[5,7])$ if there exists an $S$-map $\alpha: \mathscr{X}_{1} \otimes \mathfrak{X}_{2} \rightarrow S^{n+1}$ such that the $S$-map $\xi: \mathcal{X} \rightarrow$ $S^{n+1}$, where $\mathcal{X}=\mathfrak{X}_{1} \oplus \mathfrak{X}_{2}$, given by the matrix

$$
\left(\begin{array}{cc}
\theta_{1} & \alpha \\
(-1)^{n} \alpha^{\prime} & (-1)^{n} \theta_{2}^{\prime}
\end{array}\right)
$$

is special.

2.8. Lemma. Spherical n-pairings are contiguous if and only if the corresponding n-isometries are.

The proof is based on the following

2.9. LeMma. The n-pairing corresponding to the $n$-isometry $(\mathfrak{X}, u, z)$ is special if and only if $z^{2}=z$.

Proof. If $\mathfrak{X}=\mathfrak{X}_{1} \oplus \mathfrak{X}_{2}$ and the pairing $\theta: \mathfrak{X} \otimes \mathfrak{X} \rightarrow S^{n+1}$ is given by the matrix $\left(\begin{array}{ll}0 & v \\ 0 & 0\end{array}\right)$, where $v$ is a duality, then, as can be easily seen, the corresponding $n$-isometry $(\mathfrak{X}, u, z)$ is the following

$$
u=\left(\begin{array}{cc}
0 & v \\
(-1)^{n+1} v^{\prime} & 0
\end{array}\right), \quad z=\left(\begin{array}{cc}
0 & 0 \\
0 & 1_{x_{2}}
\end{array}\right) .
$$

But then clearly $z^{2}=z$.

Conversely, suppose we are given an $n$-isometry $(\mathcal{X}, u, z)$ satisfying $z^{2}=z$. By Theorem 1.7, there exists a virtual complex $\mathscr{Y}_{1}$ and $S$-maps $i_{1}: \mathscr{Y}_{1} \rightarrow \mathfrak{X}$ and $\pi_{1}$ : $\mathcal{X} \rightarrow \mathcal{Y}_{1}$ such that $\pi_{1} \circ i_{1}=1_{\mathscr{O}_{1}}, i_{1} \circ \pi_{1}=z$. Since $\bar{z}^{2}=\bar{z}$, we can apply Theorem 1.7 again and obtain a virtual complex $\mathscr{Y}_{2}$ and $S$-maps $i_{2}: \mathscr{Y}_{2} \rightarrow \mathfrak{X}$ and $\pi_{2}: \mathcal{X} \rightarrow \mathcal{Y}_{2}$ satisfying $\pi_{2} \circ i_{2}=1_{\mathscr{O}_{2}}, i_{2} \circ \pi_{2}=\bar{z}$. These relations imply

$$
i_{1} \circ \pi_{1}+i_{2} \circ \pi_{2}=1_{\propto} \text {. }
$$

Hence

$$
\pi_{2} \circ i_{1}=\pi_{2} \circ\left(i_{1} \circ \pi_{1}+i_{2} \circ \pi_{2}\right) \circ i_{1}=\pi_{2} \circ i_{1}+\pi_{2} \circ i_{1} \text {. }
$$

Therefore $\pi_{2} \circ i_{1}=0$ and similarly $\pi_{1} \circ i_{2}=0$.

Consider the $S$-maps $f: \mathcal{X} \rightarrow \mathcal{Y}_{1} \oplus \mathcal{Y}_{2}$ and $g: \mathscr{Y}_{1} \oplus \mathcal{Y}_{2} \rightarrow \mathfrak{X}$, where $f=\pi_{1} \oplus \pi_{2}$ and $g$ is determined by $i_{1}$ and $i_{2}$. The relations between $i_{\nu}$ and $\pi_{\nu}$, appearing in the previous paragraph, mean that $f$ and $g$ are $S$-equivalences inverse to each other. 
We have

$$
\begin{gathered}
z \circ i_{1}=i_{1} \circ \pi_{1} \circ i_{1}=i_{1}, \quad z \circ i_{2}=i_{1} \circ \pi_{1} \circ i_{2}=0, \\
u \circ\left(i_{1} \otimes i_{1}\right)=u \circ\left(z \circ i_{1} \otimes i_{1}\right)=u \circ\left(i_{1} \otimes \bar{z} \circ i_{1}\right)=0, \\
u \circ\left(i_{2} \otimes i_{2}\right)=u \circ\left(i_{2} \otimes \bar{z} \circ i_{2}\right)=u \circ\left(z \circ i_{2} \otimes i_{2}\right)=0, \\
{\left[u \circ\left(i_{1} \otimes i_{2}\right)\right]^{\prime}=(-1)^{n+1} u \circ\left(i_{2} \otimes i_{1}\right) .}
\end{gathered}
$$

Therefore $g$ defines a congruence between the given isometry and the isometry $(\mathcal{Y}, U, Z)$, where $\mathcal{Y}=\mathcal{Y}_{1} \oplus \mathcal{Y}_{2}$ and $U$ and $Z$ are given by the matrices

$$
\left(\begin{array}{cc}
0 & (-1)^{n+1} v^{\prime} \\
v & 0
\end{array}\right) \text { and }\left(\begin{array}{cc}
1_{\mathscr{O}_{1}} & 0 \\
0 & 0
\end{array}\right),
$$

respectively, where $v=(-1)^{n+1} u \circ\left(i_{2} \otimes i_{1}\right)$. Hence $v$ is a duality. The $n$-pairing corresponding to the isometry $(\mathcal{Y}, U, Z)$ is given by the matrix $\left(\begin{array}{ll}0 & 0 \\ v & 0\end{array}\right)$, as can easily be checked by direct computation, and is therefore special. This proves Lemma 2.9.

Proof of Lemma 2.8. Suppose $\left(\mathfrak{X}_{\nu}, \theta_{\nu}\right), \nu=1,2$, are contiguous spherical $n$-pairings and let $\left(X_{\nu}, u_{\nu}, z_{\nu}\right), \nu=1,2$, be the corresponding $n$-isometries. According to the definition of contiguity of pairings, there exists an $S$-map $\alpha$ : $\mathfrak{X}_{1} \otimes \mathfrak{X}_{2} \rightarrow S^{n+1}$ such that the pairing $\xi$ on $\mathfrak{X}=\mathfrak{X}_{1} \oplus \mathfrak{X}_{2}$, given by the matrix

$$
\left(\begin{array}{cc}
\theta_{1} & \alpha \\
(-1)^{n} \alpha^{\prime} & (-1)^{n} \theta_{2}^{\prime}
\end{array}\right)
$$

is special. Define the $S$-maps $\varphi: \mathfrak{X}_{1} \rightarrow \mathfrak{X}_{2}, \psi: \mathfrak{X}_{2} \rightarrow \mathfrak{X}_{1}$ by the condition

$$
u_{2} \circ\left(1_{\mathfrak{x}_{2}} \otimes \varphi\right)=(-1)^{n+1} \alpha^{\prime}=u_{1} \circ\left(\psi \otimes 1_{\mathfrak{x}_{1}}\right) \text {. }
$$

Consider the pairing $U: \mathfrak{X} \otimes \mathfrak{X} \rightarrow S^{n+1}$ and the $S$-map $Z: X \rightarrow X$ given by the matrices

$$
\left(\begin{array}{cc}
u_{1} & 0 \\
0 & -u_{2}
\end{array}\right) \text { and }\left(\begin{array}{cc}
z_{1} & \varphi \\
\psi & \bar{z}_{2}
\end{array}\right),
$$

respectively. It is easy to see that the triplet $(\mathcal{X}, U, Z)$ is an $n$-isometry and that this isometry corresponds to the pairing $\xi$. Since $\xi$ is special, Lemma 2.8 implies $Z^{2}=Z$. But the $S$-map $Z^{2}: \mathcal{X} \rightarrow \mathfrak{X}$ is given by the matrix

$$
\left(\begin{array}{ll}
z_{1}^{2}+\psi \circ \varphi & \varphi \circ z_{1}+\bar{z}_{2} \circ \varphi \\
z_{1} \circ \psi+\psi \circ \bar{z}_{2} & \bar{z}_{2}^{2}+\varphi \circ \psi
\end{array}\right) .
$$

Setting the elements of this matrix equal to the corresponding ones in the matrix defining $Z$, we get

$$
\begin{aligned}
& z_{1}=z_{1}^{2}+\psi \circ \varphi, \quad \varphi=\varphi \circ z_{1}+\bar{z}_{2} \circ \varphi, \\
& \psi=z_{1} \circ \psi+\psi \circ \bar{z}_{2}, \quad \bar{z}_{2}=\bar{z}_{2}^{2}+\varphi \circ \psi .
\end{aligned}
$$


After obvious modifications, these relations yield (II) and (III) from 2.2. The relation (I) from 2.2. follows automatically from the construction. This proves the lemma in one direction.

Conversely, suppose $\left(\mathfrak{X}_{\nu}, u_{\nu}, z_{\nu}\right), \nu=1,2$, are contiguous $n$-isometries. Suppose we are given the $S$-maps $\varphi: \mathscr{X}_{1} \rightarrow \mathfrak{X}_{2}, \psi: \mathfrak{X}_{2} \rightarrow \mathfrak{X}_{1}$ satisfying conditions (I), (II), (III) of subsection 2.2. Consider the triple ( $\mathcal{X}, U, Z$ ), where $\mathcal{X}=\mathfrak{X}_{1} \oplus \mathfrak{X}_{2}$ and the maps $U: \mathfrak{X} \otimes \mathfrak{X} \rightarrow S^{n+1}, Z: \mathscr{X} \rightarrow \mathfrak{X}$ are given by the matrices

$$
\left(\begin{array}{cc}
u_{1} & 0 \\
0 & -u_{2}
\end{array}\right) \text { and }\left(\begin{array}{cc}
z_{1} & \varphi \\
\psi & \bar{z}_{2}
\end{array}\right),
$$

respectively. An easy verification shows that this triple is an $n$-isometry. Moreover, relations (II) and (III), as above, imply $Z^{2}=Z$ and this in turn shows, by Lemma 2.8 , that the spherical pairing corresponding to the isometry $(\mathbb{X}, U, Z)$ is special. But the spherical pairing corresponding to $(\mathfrak{X}, U, Z)$ is $(\mathfrak{X}, \theta)$, where $\theta=U \circ(1$ 사 $\otimes Z$ ). A simple calculation shows that $\theta$ is given by the matrix

$$
\left(\begin{array}{cc}
\theta_{1} & \alpha \\
\beta & (-1)^{n} \theta_{2}^{\prime}
\end{array}\right),
$$

where $\theta_{1}=u_{1} \circ\left(1_{x_{1}} \otimes z_{1}\right), \quad \theta_{2}=u_{2} \circ\left(1_{\circ x_{2}} \otimes z_{2}\right), \quad \alpha=u_{1} \circ\left(1_{x_{1}} \otimes \psi\right), \quad \beta=$ $-u_{2} \circ\left(1_{x_{2}} \otimes \varphi\right)$; it follows from (I) of 2.2 that $\beta=(-1)^{n} \alpha^{\prime}$. Therefore the spherical pairings $\left(\mathfrak{X}_{1}, \theta_{1}\right)$ and $\left(\mathfrak{X}_{2}, \theta_{2}\right)$ are contiguous, but they were the ones, which corresponded to the given isometries.

The lemma is proved.

2.10. Lemma. Suppose that $\left(\mathscr{X}_{\nu}, \theta_{\nu}\right), \nu=1,2$, are two spherical $n$-pairings satisfying conn $\mathfrak{X}_{\nu} \geqslant r, \nu=1,2$. If they are $R$-equivalent, then there exists a sequence of spherical n-pairings $\left(\mathcal{Y}_{1}, \eta_{1}\right),\left(\mathcal{O}_{2}, \eta_{2}\right), \ldots,\left(\mathcal{O}_{m}, \eta_{m}\right)$, such that $\left(\mathcal{O}_{1}, \eta_{1}\right)=\left(\mathscr{X}_{1}, \theta_{1}\right)$, $\left(\mathscr{Y}_{m}, \eta_{m}\right)=\left(\mathscr{X}_{2}, \theta_{2}\right)$, for each $i$ the pairings $\left(\mathscr{\mathcal { Y }}_{i}, \eta_{i}\right)$ and $\left(\mathcal{Y}_{i+1}, \eta_{i+1}\right)$ are contiguous and also conn $\mathcal{Y}_{i} \geqslant r, i=1,2, \ldots, m$.

Proof. Since the pairings $\left(\mathfrak{X}_{1}, \theta_{1}\right)$ and $\left(\mathfrak{X}_{2}, \theta_{2}\right)$ are $R$-equivalent, there exists a sequence of spherical pairings

$$
\left(\mathscr{Z}_{1}, \xi_{1}\right),\left(\mathscr{Z}_{2}, \xi_{2}\right), \ldots,\left(\mathscr{Z}_{k}, \xi_{k}\right),
$$

such that $\left(\mathscr{Z}_{i}, \xi_{i}\right)$ and $\left(\mathscr{Z}_{i+1}, \xi_{i+1}\right)$ are contiguous for all $i$ and $\left(\mathscr{Z}_{1}, \xi_{1}\right)=\left(\mathfrak{X}_{1}, \theta_{1}\right)$, $\left(\mathscr{Z}_{k}, \xi_{k}\right)=\left(\mathcal{X}_{2}, \theta_{2}\right)$. Consider the $2 N$-fold suspensions $\Sigma^{2 N}\left(\mathscr{Z}_{i}, \xi_{i}\right)=\left(\Sigma^{2 N \mathscr{Z}_{i}}, \gamma_{i}\right)$, where $N$ is so large that for all $i$ we have the inequalities

$$
\begin{gathered}
2 N+\operatorname{conn} \mathscr{Z}_{i} \geqslant \text { length } \mathscr{I}_{i}-2, \\
3\left(2 N+\operatorname{conn} \mathscr{Z}_{i}\right) \geqslant n+4 N+1 \geqslant 6 .
\end{gathered}
$$

In view of the first one and by Corollary 1.6 we can assume, without loss of generality, that $\Sigma^{2 N \mathscr{Z}_{i}}$ is a true complex. Also using the obvious relation $\operatorname{dim} \mathscr{Z}_{i}=n$ - conn $\mathscr{Z}_{i}$, we get $\operatorname{dim} \Sigma^{2 N \mathscr{Z}_{i}}=n+2 N-$ conn $\mathscr{Z}_{i}$. We have the inequality

$$
2 \operatorname{dim} \sum^{2 N \mathscr{Z}_{i}} \leqslant 2(n+4 N) \text {. }
$$


Indeed, this inequality is equivalent to $2 N+\operatorname{conn} \mathscr{Z}_{i} \geqslant 0$, which follows from (2).

Inequality (3) implies, by the suspension theorem [18, Chapter 8] that the $S$-map

$$
\gamma_{i}: \Sigma^{2 N \mathscr{Z}_{i}} \otimes \Sigma^{2 N \mathscr{Z}_{i}} \rightarrow S^{n+4 N+1}
$$

may be presented as a continuous map which we shall denote by the same symbol. Put $r_{1}=\min _{i}\left(2 N+\operatorname{conn} \mathscr{Z}_{i}\right), n_{1}=n+4 N$.

Using Theorem 3.14 in [7] and the terminology used there, we can say that the pairings $\gamma_{1}$ and $\gamma_{k}$ are $R$-equivalent in $\mathscr{Z}_{r_{1}, n_{1}}$. But these pairings actually belong to $Z_{r+2 N, n_{1}}$ so that Theorem 3.14 in [7] immediately yields that they must be $R$-equivalent in $\mathscr{Z}_{r+2 N, n,}$ as well. Therefore, there exist $(r+2 N)$-connected complexes $\sigma_{1}, \ldots, \sigma_{l}$ and spherical pairings $\zeta_{i}: \mathcal{T}_{i} \otimes \mathcal{T}_{i} \rightarrow S^{n_{1}+1}$ such that

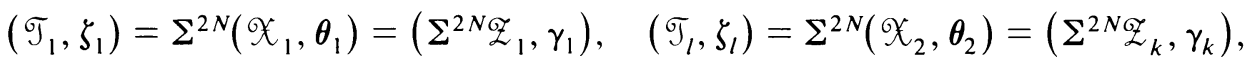

the pairings $\zeta_{i}$ and $\zeta_{i+1}$ being contiguous for all $i=1,2, \ldots, l-1$. In $\operatorname{Stab}_{0}$ we can consider the $n$-pairings $\Sigma^{-2 N}\left(\sigma_{i}, \zeta_{i}\right), i=1,2, \ldots, l$. Clearly they satisfy all the required conditions.

The lemma is proved.

2.11. The proof of Theorem 2.6 is now obtained by bringing together Theorem 3.14 in [7] with Lemmas 2.8 and 2.10. We need only use the trivial remark that any spherical $n$-pairing ( $\mathscr{X} ; \theta$ ) on a virtual complex $\mathcal{X}$ with conn $\mathfrak{X} \geqslant r$, where $3 r \geqslant n+$ $1 \geqslant 6$ is congruent to a pairing on a true $r$-connected complex presented by a continuous map. Indeed, in this case $\operatorname{dim} X \leqslant n-r$ and length $\mathcal{X} \leqslant n-2 r$. Corollary 1.6 now implies that $\mathcal{X}$ is a true complex. Further, as in the proof of Lemma $2.10, \theta$ may be presented as a continuous map.

In the sequel we shall use the following equivalent restatement of Lemma 2.10 .

2.12. Lemma. Suppose $\left(\mathfrak{X}_{\nu}, u_{\nu}, z_{\nu}\right), \nu=1,2$, are two $n$-isometries with conn $\mathfrak{X}_{\nu} \geqslant r$. If they are $R$-equivalent, then there exists a sequence of $n$-isometries $\left(\mathcal{Y}_{i}, v_{i}, z_{i}^{\prime}\right)$, $i=1,2, \ldots, m$, such that $\left(\mathcal{Y}_{1}, v_{1}, z_{1}^{\prime}\right)=\left(\mathscr{X}_{1}, u_{1}, z_{1}\right),\left(\mathcal{Y}_{m}, v_{m}, z_{m}^{\prime}\right)=\left(\mathscr{X}_{2}, u_{2}, z_{2}\right)$, for every $i$ the isometries $\left(\mathcal{Y}_{i}, v_{i}, z_{i}^{\prime}\right)$ and $\left(\mathcal{Y}_{i+1}, v_{i+1}, z_{i+1}^{\prime}\right)$ are contiguous and also conn $\mathscr{Y}_{i} \geqslant r, i=1,2, \ldots, m$.

3. Dualized spaces. In this section we consider an auxiliary homotopy problem. Here we make essential use of the results of [8].

3.1. Assume a certain integer $q \geqslant 3$ is fixed. By a dualized space, we mean a pair $(X, v)$, where $X$ is a $(q-1)$-connected finite $\mathrm{CW}$-complex with base point and $v$ : $X \wedge X \rightarrow S^{2 q+1}$ is a continuous map such that

(a) $v$ is a duality,

(b) $v^{\prime}$ is homotopic to $-v$.

Here $v^{\prime}: X \wedge X \rightarrow S^{2 q+1}$ is the composition of the map interchanging factors and $v$.

To each dualized space we now assign an algebraic object consisting of

the groups $A$ and $B$,

the homomorphism $\alpha: A \otimes \mathbf{Z}_{2} \rightarrow B$,

the forms $l: T(A) \otimes T(A) \rightarrow \mathbf{Q} / \mathbf{Z}$ and $\psi: B \otimes B \rightarrow \mathbf{Z}_{4}$.

Here $T(A)$ denotes the torsion subgroup in $A$. 
The groups $A$ and $B$ are defined as follows: $A=H_{q}(X)$ while $B=\sigma_{q+2}(X)$ is the $(q+2)$ th stable homotopy group. To define $\alpha$ consider the composition $H_{q}(X) \rightarrow$ $\pi_{q}(X) \rightarrow \sigma_{q+2}(X)$, where the first homomorphism is inverse to the Hurewicz homomorphism (which in the given case is an isomorphism), and the second one is the composition with the nontrivial element of the group $\sigma_{q+2}\left(S^{q}\right)=\mathbf{Z}_{2}$. This composition is of order 2 and thus determines a homomorphism $A \otimes \mathbf{Z}_{2} \rightarrow B$, which is taken to be $\alpha$.

Now let us define $l$. Suppose $x, y \in T(A)=$ Tors $H_{q}(X)$. Let $z \in H_{q+1}(X ; \mathbf{Q} / \mathbf{Z})$ be some class mapped into $x$ by the Bockstein homomorphism corresponding to the extension $0 \rightarrow \mathbf{Z} \rightarrow \mathbf{Q} \rightarrow \mathbf{Q} / \mathbf{Z} \rightarrow 0$. Then $l(x \otimes y)=\left\langle v^{*} s, z \wedge y\right\rangle \in \mathbf{Q} / \mathbf{Z}$, where $s \in H^{2 q+1}\left(S^{2 q+1}\right)$ is the fundamental class. Then $l$ is a well-defined form.

The form $\psi$ is defined as the composition

$$
B \otimes B=\sigma_{q+2}(X) \otimes \sigma_{q+2}(X) \rightarrow \sigma_{2 q+4}(X \wedge X) \stackrel{v}{\rightarrow} \sigma_{2 q+4}\left(S^{2 q+1}\right)=\mathbf{Z}_{24},
$$

where the first homomorphism is the exterior product. As shown in [8, §6], the group $B$ possesses an exponent which divides 4 , so that $\psi$ assumes values in $\mathbf{Z}_{4} \subset \mathbf{Z}_{24}$.

The collection $(A, B, \alpha, l, \psi)$ will be referred to as the algebraic invariant of the dualized space $(X, v)$.

Now suppose we are given two dualized spaces $\left(X_{1}, v_{1}\right)$ and $\left(X_{2}, v_{2}\right)$, and let $f$ : $X_{1} \rightarrow X_{2}$ be a continuous map. Define the continuous map $\hat{f}: X_{2} \rightarrow X_{1}$ by putting

$$
v_{2} \circ\left(1_{X_{2}} \wedge f\right) \sim v_{1} \circ\left(\hat{f} \wedge 1_{X_{1}}\right) \text {. }
$$

Since $v_{1}$ is a duality, this uniquely determines the $S$-homotopy class $\{\hat{f}\}$ and since our assumptions include the fact that $X_{1}$ and $X_{2}$ are $(q-1)$-connected and self-dual in $S^{2 q+1}$, they are homotopically $(q+1)$-dimensional, so that by the suspension theorem we have $\left[X_{2}, X_{1}\right]=\left\{X_{2}, X_{1}\right\}$ for $q \geqslant 3$. Thus $\hat{f}$ is well defined up to homotopy.

Suppose $\left(A_{\nu}, B_{\nu}, \alpha_{\nu}, l_{\nu}, \psi_{\nu}\right), \nu=1,2$, is the algebraic invariant of the dualized space $\left(X_{\nu}, v_{\nu}\right)$. The map $f$ induces homomorphisms

$$
\begin{aligned}
& A(f): A_{1}=H_{q}\left(X_{1}\right) \rightarrow A_{2}=H_{q}\left(X_{2}\right), \\
& B(f): B_{1}=\sigma_{q+2}\left(X_{1}\right) \rightarrow B_{2}=\sigma_{q+2}\left(X_{2}\right),
\end{aligned}
$$

while the map $\hat{f}$ induces the homomorphisms $A(\hat{f}): A_{2} \rightarrow A_{1}, B(\hat{f}): B_{2} \rightarrow B_{1}$. Thus to every map $f: X_{1} \rightarrow X_{2}$ we have assigned four homomorphisms $A(f), B(f), A(\hat{f})$, $B(\hat{f})$.

3.2. TheOREM. I. The algebraic invariant $(A, B, \alpha, l, \psi)$ of every dualized space $(X, v)$ satisfies the following conditions:

(a) $A$ is a finitely generated Abelian group;

(b) the sequence

$$
0 \rightarrow A \otimes \mathbf{Z}_{2} \stackrel{\alpha}{\rightarrow} B \stackrel{\beta}{\rightarrow} \operatorname{Hom}\left(A ; \mathbf{Z}_{2}\right) \rightarrow 0,
$$

where $\beta(b)(a)=\psi(b \otimes \alpha(\pi(a))), b \in B, a \in A$, and $\pi: A \rightarrow A \otimes \mathbf{Z}_{2}$ is the projection, is exact; 
(c) the pairing l is nondegenerate;

(d) the pairings l and $\psi$ are $(-1)^{q+1}$-symmetric;

(e) the composition

$$
B \stackrel{\gamma}{\rightarrow} A \stackrel{\pi}{\rightarrow} A \otimes \mathbf{Z}_{2} \stackrel{\alpha}{\rightarrow} B
$$

coincides with multiplication by 2. Here for $b \in B, \gamma^{(b)}$ is defined by the condition $\psi(b \otimes \alpha(\pi(a)))=l\left(\gamma^{(b)} \otimes a\right)$ for all $a \in T(A)$.

II. Every collection ( $A, B, \alpha, l, \psi)$ satisfying conditions (a)-(d) of statement I is the algebraic invariant of a certain dualized space.

III. Suppose $\left(A_{\nu}, B_{\nu}, \alpha_{\nu}, l_{\nu}, \psi_{\nu}\right)$ is the algebraic invariant of the dualized space $\left(X_{\nu}, v_{\nu}\right), \nu=1,2$. Let $f: X_{1} \rightarrow X_{2}$ be a continuous map. Then the homomorphisms $A(f), B(f), A(\hat{f}), B(\hat{f})$ satisfy the following conditions:

(a) the following diagrams are commutative:

$$
\begin{array}{cccccc}
A_{1} \otimes \mathbf{Z}_{2} & \stackrel{A(f) \otimes 1}{\rightarrow} & A_{2} \otimes \mathbf{Z}_{2} & A_{1} \otimes \mathbf{Z}_{2} & A(\hat{f}) \otimes 1 & A_{2} \otimes \mathbf{Z}_{2} \\
\alpha_{1} \downarrow & & \downarrow \alpha_{2} & \downarrow \alpha_{1} & & \downarrow \alpha_{2} \\
B_{1} & \overrightarrow{B(f)} & B_{2} & B_{1} & \leftarrow & B_{2}
\end{array}
$$

(b) $\psi_{1} \circ\left(B(\hat{f}) \otimes 1_{B_{1}}\right)=\psi_{2} \circ\left(1_{B_{2}} \otimes B(f)\right)$;

(c)

$$
l_{1} \circ\left(\left.A(\hat{f})\right|_{T\left(A_{2}\right)} \otimes 1_{T\left(A_{1}\right)}\right)=l_{2} \circ\left(\left.1_{T\left(A_{2}\right)} \otimes A(f)\right|_{T\left(A_{1}\right)}\right) .
$$

IV. Under the assumptions of statement III, $f \sim 0$ if and only if $A(f)=B(f)=$ $A(\hat{f})=B(\hat{f})=0$.

V. If $\left(A_{\nu}, B_{\nu}, \alpha_{\nu}, l_{\nu}, \psi_{\nu}\right)$ is the algebraic invariant of the dualized space $\left(X_{\nu}, v_{\nu}\right)$, $\nu=1,2$, then for any system of four homomorphisms $\varphi: A_{1} \rightarrow A_{2}, \xi: B_{1} \rightarrow B_{2}, \hat{\varphi}$ : $A_{2} \rightarrow A_{1}, \hat{\xi}: B_{2} \rightarrow B_{1}$ satisfying the conditions:

(a) the following diagrams are commutative:

$$
\begin{array}{cccccc}
A_{1} \otimes \mathbf{Z}_{2} & \stackrel{\varphi \otimes 1}{\rightarrow} & A_{2} \otimes \mathbf{Z}_{2} & A_{1} \otimes \mathbf{Z}_{2} & \stackrel{\hat{\varphi} \otimes 1}{\leftarrow} & A_{2} \otimes \mathbf{Z}_{2} \\
\downarrow \alpha_{1} & & \downarrow \alpha_{2} & \downarrow \alpha_{1} & & \downarrow \alpha_{2} \\
B_{1} & \vec{\xi} & B_{2} & B_{1} & \leftarrow & B_{2}
\end{array}
$$

(b) $\psi_{1} \circ\left(\hat{\xi} \otimes 1_{B_{1}}\right)=\psi_{2} \circ\left(1_{B_{2}} \otimes \xi\right)$;

(c) $l_{1} \circ\left(\left.\hat{\varphi}\right|_{T\left(A_{2}\right)} \otimes 1_{T\left(A_{1}\right)}\right)=l_{2} \circ\left(\left.1_{T\left(A_{2}\right)} \otimes \varphi\right|_{T\left(A_{1}\right)}\right)$,

there exists a map $f: X_{1} \rightarrow X_{2}$ such that

$$
A(f)=\varphi, \quad B(f)=\xi, \quad A(\hat{f})=\hat{\varphi}, \quad B(\hat{f})=\hat{\xi} .
$$

3.3. Proof of Statement I. Statement (a) is obvious. To prove (b), note that the Atiyah-Hirzebruch spectral sequence yields the exact sequence

$$
0 \rightarrow H_{q}(X) \otimes \mathbf{Z}_{2} \stackrel{\alpha}{\rightarrow} \sigma_{q+2}(X) \stackrel{\kappa}{\rightarrow} H_{q+1}\left(X ; \mathbf{Z}_{2}\right) \rightarrow 0 .
$$


The pairing $v$ defines the homomorphism

$$
\mu: H_{q+1}\left(X ; \mathbf{Z}_{2}\right) \rightarrow H^{q}\left(X ; \mathbf{Z}_{2}\right)=\operatorname{Hom}\left(A ; \mathbf{Z}_{2}\right),
$$

acting according to the formula

$$
\mu(x)(y)=v_{*}(x \wedge y) \in H_{2 q+1}\left(S^{2 q+1} ; \mathbf{Z}_{2}\right)=\mathbf{Z}_{2}, \quad y \in A .
$$

Since $v$ is a duality, $\mu$ is an isomorphism. It remains to note that $\beta=\mu \circ \kappa$, and to substitute into the exact sequence written above.

To prove (c) consider the commutative diagram

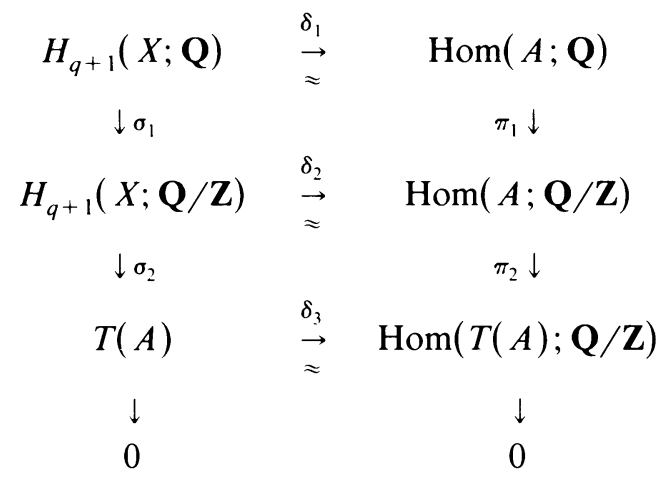

where $\sigma_{1}$ and $\pi_{1}$ are the coefficient homomorphisms, $\sigma_{2}$ is the Bockstein homomorphism, $\pi_{2}$ is the restriction homomorphism to $T(A)$ and $\delta_{1}, \delta_{2}$ act according to the formulas

$$
\begin{array}{ll}
\delta_{1}(x)(a)=v_{*}(x \wedge a), & x \in H_{q+1}(X ; \mathbf{Q}), a \in A, \\
\delta_{2}(y)(a)=v_{*}(y \wedge a), & y \in H_{q+1}(X ; \mathbf{Q} / \mathbf{Z}), a \in A .
\end{array}
$$

The homomorphism $\delta_{3}$ is well defined if we require the commutativity of this diagram. It coincides with the homomorphism associated with the form $l$. Since $v$ is a duality, $\delta_{1}$ and $\delta_{2}$ are isomorphisms. The five-by-five lemma implies that $\delta_{3}$ is an isomorphism, which implies that $l$ is nondegenerate.

Statement (d) follows from $v^{\prime} \sim-v$.

The proof of (e) will be based on statement (c) of part I of Theorem 6.3 in [8]. If we use the notations introduced there, in order to establish (e) it suffices to prove that the composition $\alpha_{41} \circ \alpha_{34}$ coincides with $\gamma$. (Here we tacitly imply that $F_{1}=A$, $F_{3}=B$.) Indeed, according to the definition of the form $l$, for $a \in T(A)$ and $b \in B$ we have $l\left(\alpha_{41}\left(\alpha_{34}(b)\right) \otimes a\right)=v_{*}\left(\alpha_{34}(b) \otimes a\right) \in H_{2 q+1}\left(S^{2 q+1} ; \mathbf{Q} / \mathbf{Z}\right)=\mathbf{Q} / \mathbf{Z}$. Clearly this coincides with $v_{*}\left(b \wedge \alpha_{13}(a)\right)=\psi\left(b \otimes \alpha_{13}(a)\right)$. But $\alpha_{13}=\alpha \circ \pi$. Hence

$$
l\left(\alpha_{41}\left(\alpha_{34}(b)\right) \otimes a\right)=\psi(b \otimes \alpha(\pi(a)))
$$

for all $a \in T(A)$ and therefore $\gamma(b)=\alpha_{41}\left(\alpha_{34}(b)\right)$ by the definition of $\gamma$. Now statement (e) follows from I.c in Theorem 6.3 (see [8]).

This proves statement $\mathrm{I}$.

3.4. Proof of statement II. We will use Theorem 7.2 of [8]. Put $F_{1}=A$, $F_{2}=\operatorname{Hom}(A ; \mathbf{Z}), F_{3}=B, F_{4}=\operatorname{Hom}(A ; \mathbf{Q} / \mathbf{Z})$. Suppose $\alpha_{13}: F_{1} \rightarrow F_{3}$ coincides with $\alpha \circ \pi$. Assume $\alpha_{34}: F_{3} \rightarrow F_{4}$ is defined by the relation $\alpha_{34}(b)(a)=\psi(b \otimes \alpha(\pi(a))) \in$ $\mathbf{Z}_{2} \subset \mathbf{Q} / \mathbf{Z}$. 
Further let $\alpha_{41}: F_{4} \rightarrow F_{1}$ be defined as follows: for $f \in \operatorname{Hom}(A ; \mathbf{Q} / \mathbf{Z})$ let $\alpha_{41}(f)$ be an element of $T(A)$ such that $l\left(\alpha_{41}(f) \otimes a\right)=f(a)$ for all $a \in T(A)$. Since the form $l$ is nondegenerate, $\alpha_{41}$ is well defined. Also define $\gamma: F_{2} \otimes \mathbf{Q} \rightarrow F_{4}$ by the formula $\gamma(g \otimes r)(a)=r g(a) \bmod \mathbf{Z}$, where $g \in F_{2}, r \in \mathbf{Q}, a \in A$.

Then, as could be easily checked, all the assumptions of statement I of Theorem 6.3 in [8] will be satisfied, so that by statement II of the same theorem there exists a finite $(q-1)$-connected complex $X$ of dimension $\leqslant q+1$ for which $F_{i}(X)=F_{i}$, $\alpha_{i j}(X)=\alpha_{i j}, \gamma(X)=\gamma$ (here we use the notations introduced in $\S 6$ of [8]).

Now define the pairings

$$
\begin{gathered}
q_{12}: F_{1} \otimes F_{2} \rightarrow \mathbf{Z}, \quad q_{21}: F_{2} \otimes F_{1} \rightarrow \mathbf{Z}, \\
q_{14}: F_{1} \otimes F_{4} \rightarrow \mathbf{Q} / \mathbf{Z}, \quad q_{41}: F_{4} \otimes F_{1} \rightarrow \mathbf{Q} / \mathbf{Z}, \quad q_{33}: F_{3} \otimes F_{3} \rightarrow \mathbf{Z}_{4} .
\end{gathered}
$$

Put $q_{12}(a \otimes f)=-q_{21}(f \otimes a)=-f(a)$, where $a \in F_{1}, f \in F_{2}$. If $g \in F_{4}, a \in F_{1}$ then $q_{14}(a \otimes g)=-q_{41}(g \otimes a)=-g(a)$. Also put $q_{33}=\psi$.

Then all the properties indicated in statement I of Theorem 7.2 in [8] are satisfied and, according to statement III of the same theorem, there exists a pairing $u$ : $X \wedge X \rightarrow S^{2 q+1}$ which induces the pairings $q_{i j}$ in the sense indicated in Theorem 7.2 of [8]. The pairing $u$ is a duality since all the conditions stated in subsection 4.5 of [8] hold. To prove $u^{\prime} \sim-u$, by statement II of Theorem 7.2 in [8] it suffices to show that $q_{i j}^{-u}=q_{i j}^{u^{\prime}}$ for all $i, j$ such that $q_{i j}$ has been defined in $\$ 7$ of [8]. But we obviously have

$$
\begin{gathered}
q_{12}^{u^{\prime}}=q_{21}^{u}, \quad q_{21}^{u^{\prime}}=q_{12}^{u}, \quad q_{14}^{u^{\prime}}=q_{41}^{u}, \quad q_{41}^{u^{\prime}}=q_{14}^{u}, \\
q_{33}^{u^{\prime}}=(-1)^{q}\left(q_{33}^{u}\right)^{\prime}=-q_{33}^{u} .
\end{gathered}
$$

On the other hand, $q_{i j}^{-u}=-q_{i j}^{u}$. By construction $q_{14}^{u}=-q_{41}^{u}, q_{12}^{u}=-q_{21}^{u}$. This implies $q_{i j}^{u^{\prime}}=q_{i j}^{-u}$ and therefore $u^{\prime} \sim-u$. Hence $(X, u)$ is a dualized space. Clearly, its algebraic invariant is the given set $(A, B, \alpha, l, \psi)$.

3.5. Proof of Statement III. The proof is quite simple. The commutativity of the diagram in statement (a) immediately follows from definition. Properties (b) and (c) follow from the relation $v_{1} \circ\left(\hat{f} \wedge 1_{X_{1}}\right) \sim v_{2} \circ\left(1_{X_{2}} \wedge f\right)$, which is the definition of $\hat{f}$.

3.6. Proof of STAtement IV. According to statement III of Theorem 6.3 in [8], to prove $f \sim 0$ it suffices to establish that $f$ induces the zero homomorphism on the functors $H_{q}, H_{q+1}, \sigma_{q+2}, H_{q+1}(; \mathbf{Q} / \mathbf{Z})$. If $A(f)=B(f)=A(\hat{f})=B(\hat{f})=0$, we have (for the first and third ones of these functors) the relations $H_{q}(f)=A(f)=0$, $\sigma_{q+2}(f)=B(f)=0$.

Suppose $a \in H_{q}\left(X_{2}\right), b \in H_{q+1}\left(X_{1}\right)$. Then

$$
\left\langle v_{2 *}\left(a \wedge f_{*}(b)\right), s\right\rangle=\left\langle v_{1 *}\left(\hat{f}_{*}(a) \wedge b\right), s\right\rangle,
$$

where $s \in H^{2 q+1}\left(S^{2 q+1}\right)$ is the generator. Since $\hat{f}_{*}(a)=A(\hat{f})(a)=0$ we have $\left\langle v_{2 *}\left(a \wedge f_{*}(b)\right), s\right\rangle=0$ for all $a \in H_{q}\left(X_{2}\right)$. In view of the fact that $v_{2}$ is a duality, it follows that $f_{*}(b)=H_{q+1}(f)(b)=0$ for all $b \in H_{q+1}\left(X_{1}\right)$.

Now suppose $a \in H_{q}\left(X_{2}\right)$ while $b \in H_{q+1}\left(X_{1} ; \mathbf{Q} / \mathbf{Z}\right)$. In view of the fact that $v_{2}$ is a duality, in order to prove that $f_{*}(b)=0$ it suffices to establish that

$$
\left\langle v_{2 *}\left(a \wedge f_{*}(b)\right), s\right\rangle=0 \in \mathbf{Q} / \mathbf{Z} \text { for all } a \in H_{q}\left(X_{2}\right) \text {. }
$$


But

$$
\left\langle v_{2 *}\left(a \wedge f_{*}(b)\right), s\right\rangle=\left\langle v_{1 *}\left(\hat{f}_{*}(a) \wedge b\right), s\right\rangle=0 \quad \text { since } \hat{f}_{*}(a)=A(\hat{f})(a)=0 .
$$

This proves statement IV.

Note that in the proof we did not make use of the fact that $B(\hat{f})=0$. Actually this condition is equivalent to the relation $B(f)=0$ by property (d) in statement III, Theorem 3.2 and the fact (which follows from statement I of the same theorem) that $\psi_{1}$ and $\psi_{2}$ are nondegenerate. We shall need this last statement later and therefore will now give its proof.

3.7. Lemma. Properties (d) and (b) from statement I of Theorem 3.2 imply that the pairing $\psi$ is nondegenerate.

Proof. Suppose $b \in B$ is an element such that $\psi\left(b \otimes b^{\prime}\right)=0$ for all $b^{\prime} \in B$. Then $\psi(b \otimes \alpha(\pi(a)))=0$ for all $a \in A$ and therefore $\beta(b)=0$. Since the sequence in (b) of Theorem 3.2 is exact, we have $b=\alpha\left(\pi\left(a_{0}\right)\right)$ for some $a_{0} \in A$. But then

$$
0=\psi\left(\alpha\left(\pi\left(a_{0}\right)\right) \otimes b^{\prime}\right)=(-1)^{q+1} \psi\left(b^{\prime} \otimes \alpha\left(\pi\left(a_{0}\right)\right)\right)=(-1)^{q+1} \beta\left(b^{\prime}\right)\left(a_{0}\right)
$$

for all $b^{\prime} \in B$. If $\pi\left(a_{0}\right) \neq 0$ then we can find a homomorphism $f \in \operatorname{Hom}\left(A, \mathbf{Z}_{2}\right)$ such that $f\left(a_{0}\right) \neq 0$. Since $\beta$ is an epimorphism, $\beta\left(b^{\prime}\right)=f$ for some $b^{\prime} \in B$. Then $\psi\left(b^{\prime} \otimes \alpha\left(\pi\left(a_{0}\right)\right)\right)=\beta\left(b^{\prime}\right)\left(a_{0}\right)=f\left(a_{0}\right) \neq 0$, which is a contradiction. Therefore $\pi\left(a_{0}\right)=0$ so that $b=0$. This means that the map

$$
\text { ass } \psi: B \rightarrow \operatorname{Hom}\left(B ; \mathbf{Z}_{4}\right)
$$

associated with $\psi$ is injective. On the other hand, (d) implies that $B$ is finite and has an exponent which divides 4 , so that the groups $B$ and $\operatorname{Hom}\left(B ; \mathbf{Z}_{4}\right)$ are of the same order. Therefore ass $\psi$ is an isomorphism.

3.8. Proof of STatement V. Assume that the homomorphisms $\varphi, \xi, \hat{\varphi}, \hat{\xi}$ possessing the assumed properties are given. To construct the map $f$ we shall use Theorem 6.3 in [8]. In particular, consider the functors $F_{i}, i=1,2,3,4$, where

$$
F_{1}=H_{q}, F_{2}=H_{q+1}, F_{3}=\sigma_{q+2}, F_{4}=H_{q+1}(; \mathbf{Q} / \mathbf{Z}) \text {. }
$$

Define the homomorphisms $\varphi_{i}: F_{i}\left(X_{1}\right) \rightarrow F_{i}\left(X_{2}\right)$ in the following way. Put $\varphi_{1}=\varphi$, $\varphi_{3}=\xi$. Define the homomorphisms $\varphi_{2}$ and $\varphi_{4}$ by the following requirements:

$$
\begin{aligned}
& q_{12}^{v_{2}} \circ\left(1_{F_{1}\left(X_{2}\right)} \otimes \varphi_{2}\right)=q_{12}^{v_{1}} \circ\left(\hat{\varphi} \otimes 1_{F_{2}\left(X_{1}\right)}\right), \\
& q_{14}^{v_{2}} \circ\left(1_{F_{1}\left(X_{2}\right)} \otimes \varphi_{4}\right)=q_{14}^{v_{1}} \circ\left(\hat{\varphi} \otimes 1_{F_{4}\left(X_{1}\right)}\right) \text {. }
\end{aligned}
$$

The constructions of the pairings $q_{i j}^{v}$ are given in $\S 7$ of [8]. Since $v_{2}$ is a duality, these requirements determine $\varphi_{2}$ and $\varphi_{4}$ entirely. By statement IV of Theorem 6.3 in [8], for the existence of a map $f: X_{1} \rightarrow X_{2}$ satisfying $F_{i}(f)=\varphi_{i}$ it is necessary and sufficient that the homomorphisms $\varphi_{i}$ commute with the natural transformations

$$
\alpha_{13}: F_{1} \rightarrow F_{3}, \quad \alpha_{34}: F_{3} \rightarrow F_{4}, \quad \alpha_{41}: F_{4} \rightarrow F_{1}, \quad \gamma: F_{2} \otimes \mathbf{Q} \rightarrow F_{4}
$$


defined in $\$ 6$ of [8]. In other words, we must check the relations

$$
\begin{aligned}
& \alpha_{13}\left(X_{2}\right) \circ \varphi_{1}=\varphi_{3} \circ \alpha_{13}\left(X_{1}\right), \\
& \alpha_{34}\left(X_{2}\right) \circ \varphi_{3}=\varphi_{4} \circ \alpha_{34}\left(X_{1}\right), \\
& \alpha_{41}\left(X_{2}\right) \circ \varphi_{4}=\varphi_{1} \circ \alpha_{41}\left(X_{1}\right), \\
& \gamma\left(X_{2}\right) \circ\left(\varphi_{2} \otimes 1_{Q}\right)=\varphi_{4} \circ \gamma\left(X_{1}\right) .
\end{aligned}
$$

The first one of them is equivalent to the commutativity of the first square in (a) of statement III of Theorem 3.2. To prove the second one, let $a \in F_{1}\left(X_{2}\right), b \in F_{3}\left(X_{1}\right)$; then

$$
\begin{aligned}
q_{14}^{v_{2}} \circ\left(a \otimes \varphi_{4} \circ \alpha_{34}\left(X_{1}\right)(b)\right) & \left.=q_{14}^{v_{1}} \circ\left(\hat{\varphi}(a) \otimes \alpha_{34}\left(X_{1}\right)(b)\right) \text { (by the definition of } \varphi_{4}\right) \\
& =q_{33}^{v_{1}} \circ\left(\alpha_{13}\left(X_{1}\right)(\hat{\varphi}(a)) \otimes b\right)(\text { by 7.2.I, (r) in [8] }) \\
& =q_{33}^{v_{1}} \circ\left(\hat{\xi}\left(\alpha_{13}\left(X_{2}\right)(a)\right) \otimes b\right)(\text { by 3.2.III }(\mathrm{a})) \\
& =q_{33}^{v_{2}} \circ\left(\alpha_{13}\left(X_{2}\right)(a) \otimes \xi(b)\right) \text { (by 3.2.V(b)) } \\
& =q_{14}^{v_{2}} \circ\left(a \otimes \alpha_{34}\left(X_{2}\right)\left(\varphi_{3}(b)\right)\right) \text { (by 7.2.I, (r) from [8]). }
\end{aligned}
$$

Since $v_{2}$ is a duality, this implies relation (4). The relations (5) and (6) are proved by a similar argument.

Thus we have shown that there exists a continuous map $f: X_{1} \rightarrow X_{2}$ such that $F_{i}(f)=\varphi_{i}, i=1,2,3,4$. Hence $A(f)=\varphi, B(f)=\xi$. The relation

$$
v_{2} \circ\left(1_{X_{2}} \wedge f\right) \sim v_{1} \circ\left(\hat{f} \wedge 1_{X_{1}}\right)
$$

implies the relations

$$
\begin{aligned}
& q_{12}^{v_{2}} \circ\left(1_{F_{1}\left(X_{2}\right)} \otimes F_{2}(f)\right)=q_{12}^{v_{1}} \circ\left(F_{1}(\hat{f}) \otimes 1_{F_{2}\left(X_{1}\right)}\right), \\
& q_{14}^{v_{2}} \circ\left(1_{F_{1}\left(X_{2}\right)} \otimes F_{4}(f)\right)=q_{14}^{v_{1}} \circ\left(F_{1}(\hat{f}) \otimes 1_{F_{4}\left(X_{1}\right)}\right) .
\end{aligned}
$$

Since $F_{2}(f)=\varphi_{2}, F_{4}(f)=\varphi_{4}$, comparing (7), (8) with (1), (2) and the fact that $v_{1}$ is a duality, we get $F_{1}(\hat{f})=A(\hat{f})=\hat{\varphi}$. Further $\psi_{1} \circ\left(\hat{\xi} \otimes 1_{B_{1}}\right)=\psi_{2} \circ\left(1_{B_{2}} \otimes \xi\right)=$ $\psi_{2} \circ\left(1_{B_{2}} \otimes B(f)\right)=\psi_{1} \circ\left(B(\hat{f}) \otimes 1_{B_{1}}\right)$ and Lemma 3.7 implies $B(\hat{f})=\hat{\xi}$.

This concludes the proof of Theorem 3.2.

4. Main result (the formulation with $P$-quintets). In this section we state and prove the main result of this paper.

Suppose $P=\mathbf{Z}[z]$ is the polynomial ring.

4.1. A $P$-quintet is defined as a collection $(A, B, \alpha, l, \psi)$ consisting of

$$
\begin{aligned}
& P \text {-modules } A \text { and } B, \\
& P \text {-homomorphism } \alpha: A \otimes_{\mathbf{Z}} \mathbf{Z}_{2} \rightarrow B, \\
& \text { the forms } l: T(A) \otimes_{\mathbf{Z}} T(A) \rightarrow \mathbf{Q} / \mathbf{Z} \text { and } \psi: B \otimes_{\mathbf{Z}} B \rightarrow \mathbf{Z}_{4}
\end{aligned}
$$

if the following conditions hold:

(a) $A$ is finitely generated by $\mathbf{Z}$;

(b) the following sequence

$$
0 \rightarrow A \otimes \mathbf{Z}_{2} \stackrel{\alpha}{\rightarrow} B \stackrel{\beta}{\rightarrow} \operatorname{Hom}_{\mathbf{Z}}\left(A ; \mathbf{Z}_{2}\right) \rightarrow 0
$$


where $\beta(b)(a)=\psi(b \otimes \alpha(\pi(a))), b \in B, a \in A$, and $\pi: A \rightarrow A \otimes \mathbf{Z}_{2}$ is the projection, is exact;

(c) the pairing $l$ is nondegenerate;

(d) pairings $l$ and $\psi$ are $\varepsilon$-symmetric;

(e) the composition

$$
B \stackrel{\gamma}{\rightarrow} A \stackrel{\pi}{\rightarrow} A \otimes \mathbf{Z}_{2} \stackrel{\alpha}{\rightarrow} B
$$

coincides with multiplication by two. Here for $b \in B, \gamma(b)$ is defined from the condition

$$
\psi(b \otimes \alpha(\pi(a)))=l(\gamma(b) \otimes a)
$$

for all $a \in T(A)$;

(f) $l(z a \otimes b)=l(a \otimes \bar{z} b), a, b \in T(A)$;

(g) $\psi(z a \otimes b)=\psi(a \otimes \bar{z} b), a, b \in B$.

The number $\varepsilon$, which may be 1 or -1 , will be called parity of the $P$-quintet.

Two $P$-quintets $\left(A_{\nu}, B_{\nu}, \alpha_{\nu}, l_{\nu}, \psi_{\nu}\right), \nu=1,2$, of the same parity are called isomorphic, if there exist isomorphisms

$$
\varphi: A_{1} \rightarrow A_{2}, \quad \xi: B_{1} \rightarrow B_{2},
$$

such that

$$
l_{1}=l_{2} \circ\left(\left.\left.\varphi\right|_{T\left(A_{1}\right)} \otimes \varphi\right|_{T\left(A_{1}\right)}\right), \quad \psi_{1}=\psi_{2} \circ(\xi \otimes \xi)
$$

and the diagram

$$
\begin{array}{ccc}
A_{1} \otimes \mathbf{Z}_{2} & \stackrel{\varphi \otimes 1}{\rightarrow} & A_{2} \otimes \mathbf{Z}_{2} \\
\downarrow \alpha_{1} & & \downarrow \alpha_{2} \\
B_{1} & \vec{\xi} & B_{2}
\end{array}
$$

is commutative.

Two $P$-quintets $\left(A_{\nu}, B_{\nu}, \alpha_{\nu}, l_{\nu}, \psi_{\nu}\right), \nu=1,2$, of the same parity are called contiguous, if there exist $P$-homomorphisms

$$
\varphi: A_{1} \rightarrow A_{2}, \quad \xi: B_{1} \rightarrow B_{2}, \quad \hat{\varphi}: A_{2} \rightarrow A_{1}, \quad \hat{\xi}: B_{2} \rightarrow B_{1}
$$

such that the following conditions hold:

(a) the following diagrams are commutative:

$$
\begin{array}{cccccc}
A_{1} \otimes \mathbf{Z}_{2} & \stackrel{\varphi \otimes 1}{\rightarrow} & A_{2} \otimes \mathbf{Z}_{2} & A_{1} \otimes \mathbf{Z}_{2} & \stackrel{\hat{\varphi} \otimes 1}{\leftarrow} & A_{2} \otimes \mathbf{Z}_{2} \\
\downarrow \alpha_{1} & & \downarrow \alpha_{2} & \downarrow \alpha_{1} & & \downarrow \alpha_{2} \\
B_{1} & \vec{\xi} & B_{2} & B_{1} & \leftarrow & B_{2}
\end{array}
$$

(b) $\psi_{1} \circ\left(\hat{\xi} \otimes 1_{B_{1}}\right)=\psi_{2} \circ\left(1_{B_{2}} \otimes \xi\right)$;

(c) $l_{1} \circ\left(\left.\hat{\varphi}\right|_{T\left(A_{2}\right)} \otimes 1_{T\left(A_{1}\right)}\right)=l_{2} \circ\left(\left.1_{T\left(A_{2}\right)} \otimes \varphi\right|_{T\left(A_{1}\right)}\right)$;

(d) the homomorphisms $\hat{\varphi} \circ \varphi, \varphi \circ \hat{\varphi}, \hat{\xi} \circ \xi, \xi \circ \hat{\xi}$ coincide with multiplications by $z \cdot \bar{z}=z(1-z) \in P$. 
The contiguity relation is reflexive and symmetric, but is not transitive. The equivalence relation generated by contiguity on the set of $P$-quintets of parity $\varepsilon$ will be called $R$-equivalence.

4.2. THEOREM. For $q \geqslant 4$ there exists a one-to-one correspondence between the set of isotopy types of $(q-1)$-simple 2q-dimensional spherical knots in $S^{2 q+2}$ and the set of $R$-equivalence classes of $P$-quintets with parity $(-1)^{q+1}$.

The remainder of this section is devoted to proving this theorem.

4.3. By Theorem 2.6, the study of $(q-1)$-simple $2 q$-dimensional knots in $S^{2 q+2}$ reduces, for $q \geqslant 4$, to the study of $2 q$-isometries on the set of virtual complexes of length $\leqslant 2$. Suppose $(\mathcal{X}, u, z)$ is such an isometry. Then conn $\mathcal{X} \geqslant q-1$ and by Corollary 1.6 we may assume, without loss of generality, that $\chi$ is a true complex. Similarly, using the ordinary suspension theorem, we may represent the $S$-map $u$ by a continuous map $\mathcal{X} \wedge \mathcal{X} \rightarrow S^{2 q+1}$, which shall be denoted by the same symbol. It now follows from the definition of isometry, given in subsection 2.1, that the pair $(\mathfrak{X}, u)$ is a dualized space. Thus, from the point of view of the notions of Theorem 3.2 , the isometry $(\mathcal{X}, u, z)$ is a dualized space $(\mathfrak{X}, u)$ supplied with a map $z$ : $\mathfrak{X} \rightarrow \mathfrak{X}$ such that $\hat{z}=1-z=\bar{z}$ (see 3.1). If we consider the algebraic invariant $(A, B, \alpha, l, \psi)$ of the dualized space $(\mathfrak{X}, u)($ see $\S 3)$, then the map $z$ defines a $P$-module structure on the groups $A$ and $B$. It follows from Theorem 3.2 that the collection $(A, B, \alpha, l, \psi)$ is a $P$-quintet of parity $(-1)^{q+1}$. Thus each $2 q$-isometry ( $X, u, z$ ), where length $\mathfrak{X} \leqslant 2$, has been assigned a certain $P$-quintet of parity $(-1)^{q+1}$.

4.4. Proposition. Every P-quintet of parity $(-1)^{q+1}$ corresponds to some $2 q$-isometry $(\mathcal{X}, u, z)$ with length $\mathfrak{X} \leqslant 2$.

Proof. First let us use statement II of Theorem 3.2 in order to construct the dualized space with a given algebraic invariant. We then apply statement $\mathrm{V}$ of Theorem 3.2, where $\varphi: A \rightarrow A$ and $\xi: B \rightarrow B$ are the multiplications by $z \in P$ while $\hat{\varphi}: A \rightarrow A$ and $\hat{\xi}: B \rightarrow B$ are the multiplications by $\bar{z}=1-z \in P$. This will give us the necessary isometry, concluding the proof.

4.5. Proposition. Two 2 -isometries on complexes of length $\leqslant 2$ are congruent (are contiguous) if and only if the corresponding P-quintets are isomorphic (respectively contiguous).

Proof. We may suppose that $q \geqslant 3$ (if not, make suspension $\Sigma^{2 N}$ for some $N$; see $\S 2)$. First consider the case of congruence. The fact that congruent isometries determine isomorphic $P$-quintets is obvious. To prove the converse, assume that we have two $2 q$-isometries $\left(\mathscr{X}_{\nu}, u_{\nu}, z_{\nu}\right), \nu=1,2$, where $\mathcal{X}_{1}$ and $\mathscr{X}_{2}$ are true complexes of length $\leqslant 2$, the $P$-quintets $\left(A_{\nu}, B_{\nu}, \alpha_{\nu}, l_{\nu}, \psi_{\nu}\right), \nu=1,2$, corresponding to these isometries being isomorphic. This means that there exist $P$-isomorphisms $\varphi: A_{1} \rightarrow A_{2}$, $\xi: B_{1} \rightarrow B_{2}$ possessing the properties indicated in the definition of quintet isomorphism (see subsection 4.1). Suppose the homomorphisms $\hat{\varphi}: A_{2} \rightarrow A_{1}, \hat{\xi}: B_{2} \rightarrow B_{1}$ are inverse to $\varphi$ and $\xi$, respectively. Then for the four homomorphisms $\varphi, \xi, \hat{\varphi}, \hat{\xi}$ the 
assumptions of statement $\mathrm{V}$ of Theorem 3.2 hold, so that there exists a continuous $\operatorname{map} f: \mathfrak{X}_{1} \rightarrow \mathfrak{X}_{2}$ satisfying

$$
A(f)=\varphi, \quad B(f)=\xi, \quad A(\hat{f})=\hat{\varphi}, \quad B(\hat{f})=\hat{\xi} .
$$

Since $\varphi$ and $\hat{\varphi}$ are isomorphisms, $f$ is a homotopy equivalence. Let us prove that $u_{1}$ is homotopic to $u_{2} \circ(f \wedge f)$. To do this, consider the map $\hat{f}: \mathfrak{X}_{2} \rightarrow \mathfrak{X}_{1}$ defined by the relation $u_{1} \circ\left(\hat{f} \wedge 1_{\mathrm{x}_{1}}\right) \sim u_{2} \circ\left(1_{\mathrm{\circ}_{2}} \wedge f\right)$. Then $A(\hat{f} \circ f)=A(\hat{f}) \circ A(f)=\hat{\varphi} \circ \varphi=$ $1_{A_{1}}$. Similarly $B(\hat{f} \circ f)=1_{B_{1}}$. Since $(\hat{f} \circ f) \sim \hat{f} \circ f$, we see that statement IV of Theorem 3.2 together with the previous relations imply $\hat{f} \circ f \sim 1_{x_{1}}$. But then

$$
\begin{gathered}
u_{1} \sim u_{1} \circ\left(\hat{f} \circ f \wedge 1_{\circ x_{1}}\right) \sim u_{1} \circ\left(\hat{f} \wedge 1_{x_{1}}\right) \circ\left(f \wedge 1_{x_{1}}\right) \\
\sim u_{2} \circ\left(1_{x_{2}} \wedge f\right) \circ\left(f \wedge 1_{x_{1}}\right)=u_{2} \circ(f \wedge f) .
\end{gathered}
$$

It remains to prove that $z_{2} \circ f \sim f \circ z_{1}$. To do this, consider the difference $z_{2} \circ f-$ $f \circ z_{1}=g$. We have

$$
A(g)=A\left(z_{2}\right) \circ A(f)-A(f) \circ A\left(z_{1}\right)=z \varphi-\varphi z=0
$$

since $\varphi$ is a $P$-homomorphism. Similarly $B(g)=0$. Moreover, $\hat{g}=\hat{f} \circ \hat{z}_{2}-\hat{z}_{1} \circ \hat{f}=$ $\hat{f} \circ \bar{z}_{2}-\bar{z}_{1} \circ \hat{f}$. Hence

$$
A(\hat{g})=A(\hat{f}) \circ A\left(\bar{z}_{2}\right)-A\left(\bar{z}_{1}\right) \circ A(\hat{f})=\hat{\varphi} \bar{z}-\bar{z} \hat{\varphi}=0
$$

since $\hat{\varphi}$ is also a $P$-homomorphism. We can show that $B(\hat{g})=0$ in a similar way. Now statement IV of Theorem 3.2 implies $g \sim 0$, so that $z_{2} \circ f \sim f \circ z_{1}$.

This proves Proposition 4.5 in the case of congruence.

Assume that the $2 q$-isometries $\left(\mathfrak{X}_{\nu}, u_{\nu}, z_{\nu}\right), \nu=1,2$, where length $\mathfrak{X}_{\nu} \leqslant 2$, have contiguous $P$-quintets $\left(A_{\nu}, B_{\nu}, \alpha_{\nu}, l_{\nu}, \psi_{\nu}\right)$. This means that there exist $P$-homomorphisms $\varphi, \xi, \hat{\varphi}, \hat{\xi}$ as in 4.1 , satisfying the conditions listed there. These conditions imply, in particular, the existence of a map $f: \mathscr{X}_{1} \rightarrow \mathfrak{X}_{2}$ such that

$$
A(f)=\varphi, \quad B(f)=\xi, \quad A(\hat{f})=\hat{\varphi}, \quad B(\hat{f})=\hat{\xi} .
$$

Also consider the map $\hat{f}: \mathscr{X}_{2} \rightarrow \mathfrak{X}_{1}$ defined by the relation $u_{1} \circ\left(\hat{f} \wedge 1_{\mathfrak{x}_{1}}\right) \sim$ $u_{2} \circ\left(1_{x_{2}} \wedge f\right)$. Since $\varphi, \xi, \hat{\varphi}, \hat{\xi}$ are module homomorphisms, by arguing as in the case of congruence, we get

$$
z_{2} \circ f \sim f \circ z_{1}, \quad z_{1} \circ \hat{f} \sim \hat{f} \circ z_{2} .
$$

Condition (d) in the definition of quintet contiguity (see subsection 4.1) implies

$$
\hat{f} \circ f \sim z_{1} \circ \bar{z}_{1}, \quad f \circ \hat{f} \sim z_{2} \circ \bar{z}_{2} .
$$

This proves that contiguity of $P$-quintets implies the contiguity of the given isometry. The converse statement is obvious.

4.6. Proof of Theorem 4.2. The proof is now immediately obtained by comparing Theorem 2.6 and Propositions 4.4 and 4.5.

\section{REFERENCES}

1. A. Dold and D. Puppe, Duality, trace, and transfer (preprint), Heidelberg, 1978.

2. D. A. Edwards and R. Geoghegan, The Wall obstruction in shape and pro-homotopy with applications, Bull. Amer. Math. Soc. 81 (1975), 919-920.

3. M. S. Farber, Linking coefficients and two-dimensional knots, Dokl. Akad. Nauk SSSR 222 (1975), 299-301; English transl., Soviet Math. Dokl. 16 (1975), 647-650. 
4. Duality in an infinite cyclic covering and even-dimensional knots, Izv. Akad. Nauk SSSR Ser. Mat. 41 (1977), 794-828; English transl., Math. USSR-Izv. 11 (1977), 749-781.

5. Classification of some knots of codimension two, Dokl. Akad. Nauk SSSR 240 (1978), 32-35. (Russian)

6. Classification of some multidimensional knots of codimension two, Uspehi Mat. Nauk 35 (1980), 105-111. (Russian)

7. I _ Isotopy types of knots of codimension two, Trans. Amer. Math. Soc. 261 (1980), 185-205.

8. Classification of stable fibred knots, Mat. Sb. 115 (1981), 223-262. (Russian)

9. P. Freyd, Splitting homotopy idempotents, Proc. Conf. Categorical Algebra (La Jolla, Calif., 1965), Springer-Verlag, Berlin and New York, 1966.

10. C. Kearton, Blanchfield duality and simple knots, Trans. Amer. Math. Soc. 202 (1975), 141-160.

11. ___ An algebraic classification of some even-dimensional knots, Topology 15 (1976), 363-373.

12. S. Kojima, A classification of some even dimensional fibred knots, J. Fac. Sci. Univ. Tokyo Sect. IA Monh. 24 (1977), 671-683.

13. Classification of simple knots by Levine pairings, Comment. Math. Helv. 59 (1979), $356-367$.

14. J. Levine, An algebraic classification of some knots of codimension two, Comment. Math. Helv. 45 (1970), 185-198.

15. __ Knot modules, Knots, Groups and 3-Manifolds, Princeton Univ. Press, Princeton, N.J., 1975, pp. 25-34.

16. Knot-modules, I, Trans. Amer. Math. Soc. 229 (1977), 1-50.

17. S. Mac Lane, Homology, Springer-Verlag, Berlin, 1963.

18. E. H. Spanier, Algebraic topology, McGraw-Hill, New York, 1971.

19. ___ Function spaces and duality, Ann. of Math. (2) 70 (1959), 338-378.

20. H. F. Trotter, On S-equivalence of Seifert matrices, Invent. Math. 20 (1973), 173-207.

BAKU, USSR 